\title{
Hepatocellular carcinoma in glycogen storage disease type IV
}

\author{
R A de Moor, J J Schweizer, B van Hoek, M Wasser, R Vink, P D Maaswinkel-Mooy
}

\begin{abstract}
A 13 year old patient with juvenile type IV glycogen storage disease died of the complications of hepatocellular carcinoma. To our knowledge this is the first reported case of hepatocellular carcinoma in association with type IV glycogen storage disease.

(Arch Dis Child 2000;82:479-480)
\end{abstract}

Keywords: glycogen storage disease type IV; hepatocellular carcinoma; arteriovenous fistula; secretory diarrhoea

Type IV glycogen storage disease (GSD; Andersen disease) is a rare autosomal recessive metabolic disorder caused by a deficiency of glycogen branching enzyme. ${ }^{1}$ Patients normally present in the first months of life with hepatosplenomegaly and failure to thrive, followed by progressive liver cirrhosis with portal hypertension. Death usually occurs before the age of 5 years. However, clinical variability has been reported. ${ }^{2-4}$

The development of a benign hepatocellular adenoma has been recognised in association with GSD IV. ${ }^{5}$ Hepatocellular carcinoma in association with GSD I, III, VI, and IX has been described. ${ }^{67}$ We report on a 13 year old boy with GSD IV and liver failure following a 10 year period with no apparent disease progression. At autopsy hepatocellular carcinoma was diagnosed.

Department of

Paediatrics, Leiden

University Medical

Centre, PO Box 9600, 2300 RC Leiden,

Netherlands

R A de Moor

J J Schweizer

P D Maaswinkel-Mooy

Department of Gastroenterology and Hepatology, Leiden University Medical Centre

B van Hoek

Department of Radiology, Leiden University Medical Centre

$\mathrm{M}$ Wasser

Department of Pathology, Leiden University Medical Centre

R Vink

Correspondence to: Dr Maaswinkel-Mooy

Accepted 3 November 1999 was found; after supplementation with L-carnitine muscle strength improved considerably $^{8}$ ).
At 13 years of age, sclerotherapy was again necessary because of haematemesis from a bleeding varix. Ultrasound revealed a small, cirrhotic liver, portal vein thrombosis, and splenomegaly.

After sclerotherapy ascites developed and diuretics were administered with good result. One month later he was readmitted because of fulminant diarrhoea with dehydration. Stool volume was 4-5 litres per 24 hours despite fasting. The stool sodium was $135 \mathrm{mmol} / \mathrm{l}$ and potassium $20 \mathrm{mmol} / \mathrm{l}$, consistent with secretory diarrhoea. Extensive examination of faecal samples yielded no infectious agent. Levels of urinary 5-hydroxyindoleacetic acid were not increased, thereby excluding carcinoid tumours. Because there was still a strong suspicion of infection, duodenoscopy and colonoscopy were performed. A notably congested bowel with mucosal oedema was seen. Biopsy specimens showed vascular ectasias, hyperaemia, and oedema. Culture of the specimens revealed no pathogen.

Symptomatic treatment with octreotide (Sandostatine) was started and the amount of diarrhoea reduced. A few days later paralytic ileus developed. Bacterial peritonitis was suspected and antibiotics were started. However, ascitic granulocyte counts were normal and cultures of ascitic fluid were negative.

The mesenterial vasculature was subject to contrast enhanced magnetic resonance angiography. Thrombosis of the portal vein was observed and a simultaneous enhancement of the intrahepatic arterial and portal system was seen, suggesting shunting between these systems. Extensive shunting between the hepatic artery and the portal vein was confirmed by digital subtraction angiography.

The patient's clinical condition and liver function deteriorated rapidly. Orthotopic liver transplantation was not performed because of the poor surgical risk. It was decided to decrease portal and intestinal venous pressure and treat the severe congestion of the bowel by occluding the hepatic artery, feeding the arteriovenous fistula, by detachable balloon embolotherapy. However, the balloon had to be removed because of hepatic failure, probably caused by ischaemia which was a result of a compromised arterial circulation. Hepatic failure progressed and the patient died three days later.

Postmortem examination revealed an enlarged cirrhotic liver (weight $1910 \mathrm{~g}$ ) with multiple nodules of hepatocellular carcinoma (fig 1) varying from $0.5 \mathrm{~cm}$ to several centimetres in diameter. There was extensive tumour necrosis. Macroscopically there was infiltrative 


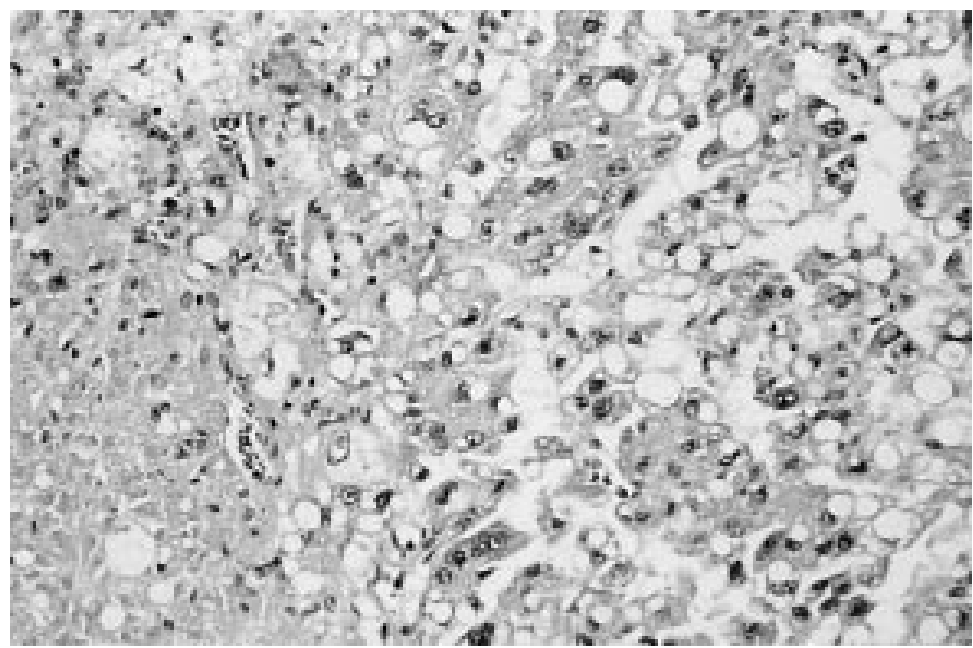

Figure 1 Partly trabecular, partly plate like hepatocellular carcinoma with necrosis (bottom left).

growth in the portal vein and subsequent thrombosis extending in mesenterial veins.

Retrospective examination of stored plasma revealed increased concentrations of $\alpha$ fetoprotein over the past three years $(128 \mu \mathrm{g} / \mathrm{l}$ in 1995 ; $33031 \mu \mathrm{g} / \mathrm{l}$ in 1997).

\section{Discussion}

We have described a 13 year old patient who died after a period of fulminant diarrhoea with dehydration and eventually liver failure, following a 10 year period with no apparent disease progression. Postmortem examination revealed hepatocellular carcinoma. To our knowledge this is the first case of hepatocellular carcinoma in a patient with GSD IV.

The classical clinical presentation for GSD IV is hepatomegaly with failure to thrive, followed by progressive liver failure and death by the age of 5 years. ${ }^{1}$ Variable expression of GSD IV has been reported. ${ }^{2-4}$ A patient, diagnosed at the age of 2 years, underwent liver transplantation at 12 years of age after an episode of shock, septicaemia, and spontaneous peritonitis. ${ }^{2}$ Guerra et al presented a girl, diagnosed at the age of 5.5 years because of feeding difficulties and hepatosplenomegaly. ${ }^{3}$ She was still doing well at the age of 8 years. McConkie-Rosell et al reported the clinical spectrum of the non-progressive juvenile form of GSD IV in four patients with long term follow up. ${ }^{4}$ None of these patients developed liver cirrhosis, skeletal muscle, cardiac, or neurological involvement. Liver enzymes returned to normal in three of the four patients before they reached 5 years of age. Our patient differs from these patients as cirrhosis and portal hypertension were already present at the age of 3 years.

Although primary malignant liver tumours account for only $0.5-2 \%$ of paediatric malignant disease, hepatocellular carcinoma has been described in GSD I, III, VI, and IX, and in other metabolic diseases such as $\alpha_{1}$ antitrypsin deficiency, tyrosinaemia, and galactosaemia. ${ }^{67}$ To date, only the association of GSD IV with a benign hepatocellular adenoma has been described. ${ }^{5}$ Because most patients with GSD IV develop liver failure before the age of 5 years, liver transplantation occurs at an early age. ${ }^{19}$ In our patient transplantation was not performed, despite cirrhosis and portal hypertension, because there had been no apparent progression of the disease over the subsequent 10 years. Few patients with GSD IV survive into their teens without treatment. We believe this may be the reason why hepatocellular carcinoma has never been described in GSD IV.

Because bleeding oesophageal varices, ascites, and diarrhoea have been associated with arterialisation of the portal circuit, ${ }^{10}$ magnetic resonance angiography and digital subtraction angiography were performed which showed arteriovenous fistulas between the hepatic artery and portal vein. The arterioportal fistulas were secondary to the hepatocellular carcinoma. The combination of an obstructed portal vein by the invasive growth of the tumour and secondary thrombosis, arterialisation of the portal circuit by arterioportal fistulas, ${ }^{10}$ and variceal sclerotherapy ${ }^{11}$ will have increased portal blood flow and pressure considerably. We suggest that the raised portal pressure resulted in secretory diarrhoea and ascites.

Hepatocellular carcinoma is a possible complication in GSD IV. We advise that patients with GSD IV are screened for hepatocellular carcinoma by regular assessment of serum $\alpha$ fetoprotein and ultrasound, for example, twice a year.

1 Chen YT, Burchell A. Glycogen storage diseases. In: Scriver CR, Beaudet AL, Sly WS, Valle D, editors. The metabolic and molecular bases of inherited disease. New York: McGraw Hill, 1995:935-65.

2 Dhawan A, Tan KC, Portmann B, Mowat AP. Glycogenesis type IV: liver transplant at 12 years. Arch Dis Child 1994;71:450-1.

3 Guerra AS, van Diggelen OP, Carneiro F, Tsou RM, Simoes S, Teixeira Santos N. A juvenile variant of glycogenosis IV (Andersen disease). Eur f Pediatr 1986;145:179-81.

4 McConkie-Rosell A, Wilson C, Piccoli DA, et al. Clinical and laboratory findings in four patients with the nonprogressive hepatic form of type IV glycogen storage progressive hepatic form of type IV glyc

5 Alshak NS, Cocjin J, Podesta L, et al. Hepatocellular adenoma in glycogen storage disease type IV. Arch Pathol Lab Med 1994;118:88-91.

6 Mowat AP. Liver tumours. In: Liver disorders in children, 3rd edition. Oxford: Butterworth-Heinemann Ltd, 1994:398412.

7 Haagsma EB, Smit GPA, Niezen-Koning KE, Gouw ASH, Meerman L, Slooff MJH, and the liver transplant group. Type IIIb glycogen storage disease associated with end-stage cirrhosis and hepatocellular carcinoma. Hepatology 1997;25:537-40.

8 Maaswinkel-Mooy PD, Poorthuis BJHM, Van Gelderen $\mathrm{HH}$, Van de Kamp JJP. Dicarboxylicaciduria and secondary carnitine deficiency in glycogenosistype IV. Arch Dis Child 1987;62:1066-7.

9 Selby R, Starzl TE, Yunis E, Brown BI, Kendall RS, Tzakis A. Liver transplantation for type IV glycogen storage disease. N Engl F Med 1991;324:39-42.

10 Galler G, Yim A, Emond JC, Kirch E, Yousefzadeh D, Baker AL. Splenic arteriovenous fistula: a rare lesion causing bleeding esophageal varices, ascites, and diarrhea. Dig Dis Sci 1996;41:816-19.

11 Shimoji K,Itoh S, Kameda C, Shiomi M. ${ }^{99 \mathrm{~m}}$ Tc-DTPA activity in the azygos vein before and after sclerotherapy in patients with cirrhosis. Eur F Radiol 1993;17:106-12. 